\title{
Pharmacovigilance in Hospice/Palliative Care: De-Prescribing Combination Controlled Release Oxycodone-Naloxone
}

\author{
Katherine Clark, MBBS, MMED, PhD, FRACP, FAChPM,1-3 Paul G. Byrne, BSc Masters Biostats,2 \\ Jane Hunt, RN, ${ }^{3}$ Linda Brown, MBus, BBus (Mgmt), ${ }^{3}$ \\ Debra Rowett, BPharm, Adv Prac Pharm, FPS, MSHPA, ${ }^{4}$ Gareth Watts, FAChPM, ${ }^{5}$ \\ Melanie Lovell, BBS, PhD, FRACP, FAChPM, 2,3 \\ and David C. Currow, BMed, MPH, PhD, FRACP, FAHMS ${ }^{3}$
}

\begin{abstract}
Background: Pharmacovigilance studies in hospice/palliative care provide extra information to improve medication safety. Combination controlled release oxycodone-naloxone offers an alternative opioid with less risk of opioid-induced constipation.

Objective: To examine why palliative care clinicians chose to cease oxycodone-naloxone and to explore immediate and short-term benefits and harms of this medication change.

Design: A consecutive cohort study.

Setting: 112 adults from 13 palliative care centers.

Measurements: Reasons for ceasing medication and the harms and benefits that followed this 24 and 72 hours later. Symptom burdens were summarised by the National Cancer Institute Common Terminology Criteria for Adverse Events Toxicity Gradings.

Results: Combination medication was most commonly ceased because of poor pain control or impaired hepatic function. The last median oral morphine equivalent oxycodone dose before the switch was $45 \mathrm{mg}$ (range 7.5$240 \mathrm{mg}$ ) with 76 switched to an alternative long-acting opioid (initial median oral morphine equivalent dose being $45 \mathrm{mg}$ [range 5-210 mg]). Subgroup analysis of those switched because of clinicians' concerns about hepatic dysfunction demonstrated this group were receiving significantly lower opioid doses pre-cessation compared to those switched because of other reasons $(p=0.007)$. Regardless of why the medication was changed, improvements in pain and constipation scores were seen, the latter associated with an attendant increase in laxatives.

Conclusions: This preliminary work suggests that despite theoretical concerns regarding the effect of the naloxone on opioid doses, most people were switched safely to very similar opioid doses with attendant improvements in pain control.
\end{abstract}

Keywords: opioid-induced constipation; opioids; pharmacovigilance

\section{Background}

$\mathbf{P}$ AIN REMAINS a SIGNIFICANT ISSUE for people with lifelimiting illnesses. Estimates suggest that up to $90 \%$ of people with advanced cancer and $80 \%$ with nonmalignant life-limiting illnesses are likely to experience severe pain ${ }^{1,2}$ requiring opioid analgesia. ${ }^{3}$ Although opioids are very effective agents for strong pain, they are also associated with predictable harms including constipation. ${ }^{4}$ Changes in bowel habits as the result of opioids are common and often

\footnotetext{
${ }^{1}$ Department of Palliative Care, Northern Sydney Local Health District Cancer and Palliative Care Network, St. Leonards, Australia.

${ }^{2}$ Health Sciences, Northern Clinical School, The University of Sydney, Sydney, Australia.

${ }^{3}$ School of Medicine and Public Health, IMPACCT (Improving Palliative, Aged and Chronic Care through Clinical Research and Translation), University of Technology Sydney, Australia.

${ }^{4}$ School of Pharmacy and Medical Sciences, University of South Australia, Adelaide, Australia

${ }^{5}$ The University of Newcastle, Newcastle, Australia.

Accepted October 28, 2019.
} 
persistent despite usual interventions, and failure to adequately address the symptoms of constipation can impair quality of life. ${ }^{5}$

The combination preparation of controlled release (CR) oxycodone with naloxone offers an alternative to usual opioids when strong analgesia is indicated. However, compared with other opioids, the combination medication confers less risk of opioid-induced constipation. The efficacy of naloxone with oxycodone has been examined in a number of quality trials and reviews in both malignant and nonmalignant pain. ${ }^{6-16}$ These data are sufficiently robust to permit registration of combination oxycodone-naloxone by a number of regulatory agencies, including the Australian Pharmaceutical Benefits Scheme.

Oral naloxone's effect on bowel function is mediated through competitive opioid antagonism in the gut wall. Naloxone is well absorbed orally but then undergoes extensive first-pass hepatic metabolism ( 97-98\%) resulting in low systemic bioavailability. This means that analgesia is not compromised even though there has been antagonism of intestinal opioid receptors. ${ }^{17}$ While this is an attractive solution to bowel problems, many palliative care patients are at risk of prolonged first pass metabolism due to changes in hepatic enzyme activity, plasma protein and blood cell binding, and gastrointestinal motility. ${ }^{18}$ This can lead to a reduced analgesic effect of the oxycodone due to increased systemic availability of naloxone. This concern has been raised in a number of case reports where clinicians have switched patients with impaired liver function from equipotent doses in the combination medication to oxycodone alone with this change resulting in opioid toxicity. ${ }^{19,20}$ Aside from the concerns around altered first pass metabolism, there are other issues that require further examination, one of which is that clinical observations suggest that some people receiving the combination medication continue to experience distressing bowel problems and still require additional laxatives. ${ }^{21}$

This pragmatic, consecutive cohort study was conducted with the aim of documenting why clinicians choose to cease combination oxycodone-naloxone and when they do, how they then manage their patient's pain and constipation symptoms. This was done to detect whether signals could be identified that might suggest a possible causal relation between adverse events (AEs) and the medication that have not been previously explored and therefore require further investigation.

The work presented here is part of an international pharmacovigilance project that commenced in $2011 .^{22}$ This program collects data prospectively, documenting the immediate and short-term net clinical effects (benefits and harms) of medications commonly used for symptom control in palliative care. Already several studies have been published that describe the clinical experiences of patients when prescribed medications in routine clinical practice. ${ }^{23-26}$ This article builds on that work but extends the program to examine deprescribing $\mathrm{CR}$ combination oxycodone with naloxone and the change to alternative long-acting opioids.

\section{Methods}

Full details of the methods of the pharmacovigilance program have been described previously. ${ }^{22}$ However, unlike other RAPID studies so far completed, this work was undertaken with the aim of documenting clinical outcomes of de-prescribing. Data were collected when a decision to cease combination oxycodone-naloxone $\mathrm{CR}$ was reached as part of routine clinical care. Along with anonymized patient clinical and demographic data, the reasons for ceasing combination $\mathrm{CR}$ oxycodone-naloxone were collected and entered into a secure website (www.caresearch.com.au) customized for this work. The reasons selected for de-prescribing were nominated by the researchers based on their clinical reasoning and experience when working with the combination medication. Other data collected included the following: the daily dose of oxycodone in the combination medication prescribed on the day it was ceased; whether there were adverse symptoms of interest relating to opioids at baseline; the alternative opioid selection including whether this was CR or immediate release (IR); and whether symptoms changed from baseline.

Data were collected at baseline (T0; the day the combination medication was ceased) then again 24 (T1) and 72 hours later (T2).

\section{Symptom scores}

Symptom scores including pain, nausea, drowsiness, confusion, and myoclonus were summarized using the widely used National Cancer Institute Common Terminology Criteria for Adverse Events (NCI CTCAE) toxicity gradings. ${ }^{27}$ The NCI CTCAE uses descriptive terminology to summarize severity assessments of AEs as listed here: Grade 1-Mild AE, Grade 2-Moderate AE, Grade 3-Severe AE, Grade 4-Life-threatening or disabling AE, and Grade 5-Death related to AE. Overall benefit was defined as a one-point reduction, in line with other work undertaken by this group.

\section{Other variables of interest}

In addition to NCI toxicity criteria, other constipation assessments included the following questions: is this patient constipated (yes/no); is this patient on laxatives (yes/no); and has this patient had a bowel action in the last three days (yes/ no). People's medication charts were examined to extract laxatives used. Laxatives prescribed at baseline were documented and compared with laxatives taken at T1 and T2.

The final item of interest was whether there was any difference in the subsequent opioid dose that clinicians ordered when the combination medication was switched because of concerns about liver dysfunction compared to other reasons for switching.

\section{Statistical analysis}

All data analysis was performed using Stata/IC V13.1 (c) StataCorp LP, College Station, TX. 2013).

Comparisons of symptom severity scores at the three time points were performed using Wilcoxon matched-pairs signedrank tests. When scores were documented as ungradable, they were omitted from the analysis. The case report form did not allow pain scores to be given as "No symptom" at baseline, whereas that score was an option at the later time points. A sensitivity analysis was performed to confirm that this variation in available scores had not skewed the test outcomes. The comparisons were repeated using two alternative treatments 
Table 1. Demographic Details $(N=112)$

\begin{tabular}{lc} 
Variable & Summary statistics \\
\hline Median age (range) & $74.5(26-97)$ \\
Number. of men compared to women & $56: 56$ \\
Median BMI (range) & $23.4(16.0-42.0)$ \\
Australia-modified Karnofsky & $n(\%)$ \\
Performance Scale & \\
$\geq 80$ & $6(5.4)$ \\
60-70 & $24(21.5)$ \\
$40-50$ & $57(50.9)$ \\
20-30 & $25(22.3)$ \\
Primary life-limiting illness & $n(\%)$ \\
Metastatic cancer & $107(95.5)$ \\
Renal failure & $1(0.9)$ \\
Cardiac failure & $1(0.9)$ \\
Neurodegenerative disease & $1(0.9)$ \\
Other & $2(1.8)$ \\
Mean weighted Charlson comorbidity & $6.4 \pm 3.0$ \\
index score \pm SD & \\
Pathology (where available) & \\
Serum albumin $(n=89)$ & Mean \pm SD \\
INR $(n=40)$ & $28.5 \pm 7.0$ \\
C-reactive protein $(n=50)$ & $1.2 \pm 0.3$ \\
Calculated creatinine clearance & $72.7 \pm 77.1$ \\
or eGFR $(n=92)$ & $79.3 \pm 32.0$ \\
Hemoglobin $(n=94)$ & $106.5 \pm 24.2$ \\
\hline
\end{tabular}

${ }^{\mathrm{a}}$ This score omitted the primary life-limiting illness.

BMI, body mass index; eGFR, estimated Glomerular Filtration Rate; INR, International Normalized Ratio; SD, standard deviation.

of the data, first omitting patients with baseline scores of 1 , and second by adjusting baseline scores of 1 to be 0 . The other symptoms did not require similar treatment.

The comparisons between time points of the dichotomous outcomes were performed using symmetry exact tests. Wilcoxon matched-pairs signed-rank tests were used to perform the comparisons of the number of different laxatives being prescribed.

Mann-Whitney $U$ tests were used to compare the pain scores, changes in pain scores, CR opioid doses, and changes in CR opioid dose between the group who ceased combination oxycodone-naloxone for reasons of impaired liver function, and the group of all other patients.

No adjustment was made for multiple tests as the results were considered to be hypothesis generating.

\section{Results}

This study used data collected from 112 patients from 12 Australian sites and 1 international site. At 24 hours, data were entered for 108 patients and for 100 at 72 hours. The characteristics of the group at baseline are summarized in Table 1.

\section{Reasons given for ceasing combination oxycodone-naloxone}

Clinicians were asked to nominate the main reason that they chose to switch from CR oxycodone-naloxone with their choices including the following: poor pain control in $43.8 \%$ $(n=49)$; impaired liver function in $41.1 \%(n=46)$; adverse drug reaction in $3.6 \%(n=4)$; inability to tolerate oral medications in $4.5 \%(n=5)$; and other reasons (not specified) in $7.1 \%(n=8)$.

\section{Opioid use}

At baseline, 112 had been receiving combination oxycodone with naloxone, with dosages available for 105 and median oral morphine equivalent dose (OMED) $45 \mathrm{mg}$ (range $7.5-240 \mathrm{mg}$ ). Following cessation of the combination medication, $67.8 \%(n=76)$ were switched to an alternative CR opioid with the initial median OMED (Table 2) being $45 \mathrm{mg}$ (range 5-210). Of the remaining patients, 26.8\% $(n=30)$ were commenced on short-acting opioids and $5.4 \%(n=6)$ were not given opioids at all.

\section{Pain scores}

Pain scores were assessed at baseline and then again at T1 and T2. The median pain score at baseline $(n=111)$ was 2 (IQR: $2-3)$. At T1 $(n=106)$ the median pain score was 2 (IQR: 1-2), with improvement being recorded for $51.9 \%$ $(n=55)$, deterioration for $6.6 \%(n=7)$, and no change seen in $41.5 \%(n=44)$ of the patients, compared to baseline $(p<0.001)$. Median score at T2 $(n=99)$ was 2 (IQR: $1-2)$, with $57.6 \%(n=57)$ of patients improving and $3.0 \%(n=3)$ declining compared to baseline $(p<0.001)$. In $39.4 \%(n=39)$ there was no change. The sensitivity analysis demonstrated that the highly significant differences between pain scores at baseline (see Supplementary Data) and the other time points still held in the alternative methods.

Table 2. Alternative Long-Acting Opioids $(N=76)$

\begin{tabular}{lcccccc}
\hline Medication & $\mathrm{n}$ & $\begin{array}{c}\text { Conversion } \\
\text { factor }\end{array}$ & $\begin{array}{c}\text { Median opioid } \\
\text { dose per 24 hours }\end{array}$ & Range & $\begin{array}{c}\text { Median OMED } \\
(\text { mg) }\end{array}$ & Range (mg) \\
\hline Buprenorphine & 5 & 75 & $240 \mu \mathrm{g}$ & $120-720 \mu \mathrm{g}$ & 18 & $9-54$ \\
Fentanyl & 4 & 100 & $740 \mu \mathrm{g}$ & $290-1200 \mu \mathrm{g}$ & 74 & $29-120$ \\
Hydromorphone & 7 & 5.0 & $12 \mathrm{mg}$ & $4-40 \mathrm{mg}$ & 60 & $20-200$ \\
Methadone & 1 & 1.0 & $200 \mathrm{mg}$ & -500 & $5-80$ \\
Morphine & 10 & 1.0 & $20 \mathrm{mg}$ & $5-80 \mathrm{mg}$ & 52.5 & $15-210$ \\
Oxycodone & 48 & 1.5 & $200 \mathrm{mg}$ & $10-140 \mathrm{mg}$ & 66.7 & - \\
Tapentadol & 1 & 0.33 & - & &
\end{tabular}

OMED, oral morphine equivalent dose. 
Table 3. Patients with Hepatic Dysfunction Versus All Others

\begin{tabular}{|c|c|c|c|c|c|}
\hline \multirow[b]{2}{*}{ Age, median (range) } & \multicolumn{2}{|c|}{ Hepatic dysfunction } & \multicolumn{2}{|r|}{ All others } & \multirow{2}{*}{$\begin{array}{c}\mathrm{P} \\
0.43\end{array}$} \\
\hline & $n=46$ & $\begin{array}{c}74.5(26 \text { to } 89) \\
23: 23\end{array}$ & $n=66$ & $\begin{array}{c}74.5(38 \text { to } 97) \\
33 \cdot 33\end{array}$ & \\
\hline BMI, median (range) & $n=34$ & 23.9 (18.6 to 42.0$)$ & $n=46$ & $23.2(16.0$ to 37.8$)$ & 0.12 \\
\hline \multicolumn{6}{|l|}{ Pain scores, median (IQR) } \\
\hline Baseline & $n=46$ & $2(2$ to 3$)$ & $n=65$ & $2(2$ to 3$)$ & 0.04 \\
\hline 24 hours & $n=44$ & $1.5(1$ to 2$)$ & $n=62$ & $2(1$ to 3$)$ & 0.05 \\
\hline Mean change in 24 hours & $n=44$ & -0.6 (SD: 0.12$)$ & $n=62$ & -0.6 (SD: 0.12$)$ & 0.58 \\
\hline 72 hours & $n=41$ & $1(1$ to 2$)$ & $n=58$ & $2(1$ to 2$)$ & $<0.001$ \\
\hline Mean change in 72 hours & $n=41$ & -0.9 (SD: 0.12) & $n=58$ & -0.7 (SD: 0.12$)$ & 0.08 \\
\hline \multicolumn{6}{|l|}{ Daily OMED, median (range) } \\
\hline Baseline & $n=43$ & $30(15$ to 120$)$ & $n=62$ & $60(7.5$ to 240$)$ & 0.007 \\
\hline After switching & $n=38$ & $30(10$ to 120$)$ & $n=37$ & $60(5$ to 210$)$ & 0.008 \\
\hline Change & $n=35$ & $0(-40$ to 90$)$ & $n=35$ & $0(-165$ to 170$)$ & 0.64 \\
\hline
\end{tabular}

IQR, InterQuartile Range.

\section{Comparison of those who ceased oxycodone because of concern of liver dysfunction with all others}

A subgroup comparison was undertaken to compare the differences between those switched from the combination medication to another long-acting opioid due to concerns regarding liver dysfunction compared to other patients. The results summarized in Table 3 demonstrate that in terms of age, sex, and body mass index, the groups were similar.

When the CR medication OMED at baseline was compared between groups, the baseline OMED in the hepatic dysfunction group $(n=43)$ was significantly lower than others $(n=62 ; p=0.007)$. This was also true when comparing doses of CR opioids after switching for the two groups $(p=0.008)$. When the CR OMED doses that people were switched to were compared to their baseline doses, the median dose change in each group was 0 .

\section{Constipation, bowel scores, and laxative use}

At baseline and then again at $\mathrm{T} 1$ and $\mathrm{T} 2$, as per usual practice, constipation was documented as present or absent as summarized in Table 4. Other clinical data recorded whether a bowel action had occurred within three days, whether laxatives were

Table 4. Clinicians Assessment of Constipation at Each Time Point

\begin{tabular}{|c|c|c|c|}
\hline \multirow[b]{2}{*}{$\begin{array}{l}\text { Clinical assessment } \\
\text { of constipation }\end{array}$} & \multicolumn{3}{|c|}{ Yes (\%) } \\
\hline & $\begin{array}{c}\text { T0 } \\
\text { (baseline) } \\
\mathrm{n}=112\end{array}$ & $\begin{array}{c}T 1 \\
(24 \text { hours }) \\
\mathrm{n}=108\end{array}$ & $\begin{array}{c}T 2 \\
(72 \text { hours }) \\
\mathrm{n}=100\end{array}$ \\
\hline $\begin{array}{l}\text { Is this person } \\
\text { constipated? }\end{array}$ & $58(51.8)$ & $43(40.2)^{\mathrm{a}}$ & $44(44.4)^{\mathrm{a}}$ \\
\hline $\begin{array}{l}\text { Is this person on } \\
\text { laxatives? }\end{array}$ & 89 (79.5) & $92(85.2)$ & $85(85.0)$ \\
\hline $\begin{array}{l}\text { Has this person had } \\
\text { a bowel action } \\
\text { within three days? }\end{array}$ & $90(81.1)^{\mathrm{a}}$ & $90(84.9)^{b}$ & $76(78.4)^{\mathrm{c}}$ \\
\hline
\end{tabular}

prescribed, and the severity of constipation. Additionally, all the laxatives being administered at each time point were recorded.

Compared with baseline, clinicians identified fewer of their patients as constipated at T1 and T2. Between T0 and T1 the number of patients who changed from constipated to not constipated $(n=18)$ was significantly more than the shift in the other direction $(n=5 ; p=0.01)$. Between T0 and T2 the result was less significant $(p=0.12)$. Comparisons of the numbers of patients taking laxatives did not show significant differences between the baseline and later time points.

Only marginal changes were noted in the NCI constipation severity scores. At baseline the median score was 1 (IQR: 0-2). Identical median and IQR scores were recorded at T1, with 15 patients improving and 5 deteriorating compared to baseline $(p=0.02)$. At T2, the median score was 0.5 (IQR: $0-2)$, with 24 patients improving and 12 declining compared to baseline $(p=0.03)$.

As seen in Table 5, there was a shift toward higher numbers of laxatives being administered between T0 and T2 $(p=0.002)$.

\section{Other symptoms at baseline and post-switching}

Nausea scores at T1 improved in 26 but declined in 6 compared to baseline $(p<0.001)$. At T2, nausea improved in 25 but deteriorated in 6 compared to baseline $(p<0.001)$. Drowsiness scores improved in 7 but deteriorated in 17 between baseline and T1 $(p=0.05)$. However, no significant change was subsequently seen between baseline and T2 $(p=0.17)$, so the earlier difference appears to have been temporary. Less than $30 \%(n=29)$ were confused at baseline with no change in this problem between baseline and later time points.

\section{Table 5. Numbers of Standard Laxatives Being Prescribed to How Many Patients}

\begin{tabular}{lccc}
\hline $\begin{array}{l}\text { No. of standard } \\
\text { laxatives per } \\
\text { patient }\end{array}$ & $\begin{array}{c}\text { T0 } \\
\text { (baseline) } \\
\mathrm{n}(\%)\end{array}$ & $\begin{array}{c}\text { T1 } \\
(24 \text { hours) } \\
\mathrm{n}(\%)\end{array}$ & $\begin{array}{c}\text { T2 } \\
\text { (72 hours) } \\
\mathrm{n}(\%)\end{array}$ \\
\hline 0 & $23(21)$ & $16(15)$ & $14(14)$ \\
1 & $50(45)$ & $46(43)$ & $39(39)$ \\
2 & $30(27)$ & $33(31)$ & $31(31)$ \\
3 & $8(7)$ & $9(8)$ & $10(10)$ \\
4 & $1(1)$ & $4(4)$ & $6(6)$ \\
\hline
\end{tabular}




\section{Discussion}

The aim of this pragmatic, consecutive-cohort pharmacovigilance study was to examine the de-prescribing of CR combination oxycodone and naloxone in real clinical situations. In the vast majority of cases, this occurred because of inadequate pain control, or concerns about the safety of the medication when liver function is impaired. Those who were switched because of poor pain control were typically on much higher doses than those switched because of concern regarding hepatic dysfunction. While some of the participants were changed to short-acting opioids, most were switched to long-acting. When the pre-switching opioid doses of CR combination medication were compared with the post-switching CR opioids, little corresponding change in the 24-hour equivalent dose of morphine was noted.

This was a surprising find for three reasons. First, guidelines recommend that opioid doses are reduced when moving from one opioid to another. ${ }^{28}$ This is due to possible interactions of opioids and opioid receptors including incomplete crosstolerance and intra-individual variability. ${ }^{29}$ Second, the wide variations quoted in dose conversion tables ${ }^{30}$ have led to some suggestions that when switching, the safest practice might even be to introduce the next medication at an opioid-naïve starting dose. ${ }^{31}$ Third, while there is little available data to guide opioid switching when the initial opioid is combined with an opioid antagonist, the combination medication is not recommended in hepatic dysfunction because of the possible prolongation of naloxone's half-life. Such prolongation can result in an effective reduction in the oxycodone dose, which suggests that an opioid switch should be made to a lower dose of the new longacting opioid. In this observational study, the baseline and subsequent doses were overall very similar even in people where hepatic dysfunction was cited as the reason for change.

For the most part, the change to alternative long-acting opioids appeared to have been well tolerated with significant improvements in pain scores. In addition, constipation scores improved. The main clinical benefit of the combination medication is reduced opioid-induced constipation. It is a highly relevant finding that this work suggests that a switch from combination oxycodone-naloxone to an alternative agent with an associated increase in laxatives actually improved constipation scores. This observation requires further prospective research. Opioids contribute to constipation through binding on the $\mu$-receptor in the intestine, but this is unlikely to be the only mechanism for constipation in palliative care. Factors such as reduced performance status, high anticholinergic burden, and altered oral intake are likely to be contributing factors. ${ }^{32}$ It is possible that the combination medication reduces the vigilance with which this symptom is addressed and the switch prompted a review of constipation leading to an increase in laxatives. A key question for the future is whether constipation might be more tolerable when people receive the combination medication with usual laxatives compared with oxycodone plus usual laxatives.

\section{Strengths and limitations}

This phase IV study was conducted to examine real clinical practice in palliative care. While this is a strength of the work it is also a weakness because detailed data interrogation was not possible. The details of the degree of hepatic dysfunction were not included and hence it is not possible to understand whether clinicians were concerned that liver function would deteriorate in the future or if it was currently poor. Unlike other palliative care studies conducted by this group, the data for this work were mostly collected from participating sites in Australia with only one international location. It is not clear whether the practices summarized here are reflective of how oxycodone-naloxone is used more globally or if the concerns expressed by Australian doctors are a peculiar local issue. The majority of people included in the study had a cancer diagnosis accounting for their referral to palliative care with very few with nonmalignant diagnoses. All were included as this was a pragmatic project, but this fact limits the generalizability of the results. The majority of people were switched from $\mathrm{CR}$ oxycodone with naloxone to $\mathrm{CR}$ oxycodone alone. However, there were some who switched to other longacting preparations and some to IR opioids. These observations raise two comments and also limit the generalizability of this work. Of those switched to long-acting opioids, most were switched to oxycodone alone suggesting that the main change was the cessation of naloxone. In future work, the experiences of people switched to other opioids such as morphine will require detailed exploration. The other group was those that switched to short-acting opioids. It is possible that this group may have been switched to lower doses, but this detail was not available. Lastly, constipation is a highly subjective symptom and any further work needs to include people's self-reports of their bowel symptoms.

The strengths of this work are that it builds on other phase IV studies but adds a new dimension-de-prescribing. Further, work such as this is important since it aims to explore issues raised by clinicians when prescribing medications that have been introduced to routine clinical practice based on phase III efficacy studies. Without such work, it is not possible to understand these concerns with this information invaluable to inform future investigations.

\section{Acknowledgment}

The authorship team would like to acknowledge all the participating sites including the patients they care for and the staff who provide this care.

\section{Funding Information}

The authors received no financial support for the research, authorship, or publication of this article.

\section{Author Disclosure Statement}

No competing financial interests exist.

\section{Supplementary Material}

Supplementary Data

\section{References}

1. Solano JP, Gomes B, Higginson IJ: A comparison of symptom prevalence in far advanced cancer, AIDS, heart disease, chronic obstructive pulmonary disease and renal disease. J Pain Symptom Manage 2006;31:58-69.

2. Murtagh FE, Preston M, Higginson I: Patterns of dying: Palliative care for non-malignant disease. Clin Med 2004;4:39-44.

3. Gao W, Gulliford M, Higginson IJ: Prescription patterns of analgesics in the last 3 months of life: A retrospective 
analysis of 10202 lung cancer patients. Br J Cancer 2011; 104:1704-1710.

4. Committee EG, Fallon M, Giusti R, et al.: Management of cancer pain in adult patients: ESMO clinical practice guidelines. Ann Oncol 2018;29(Supplement_4):iv166-iv191.

5. Candy B, Jones L, Goodman ML, et al.: Laxatives or methylnaltrexone for the management of constipation in palliative care patients. Cochrane Database Syst Rev 2011:CD003448.

6. Huang L, Zhou JG, Zhang Y, et al.: Opioid-induced constipation relief from fixed-ratio combination prolongedrelease oxycodone/naloxone compared with oxycodone and morphine for chronic nonmalignant pain: A systematic review and meta-analysis of randomized controlled trials. J Pain Symptom Manage 2017;54:737-748.e733.

7. Blagden M, Hafer J, Duerr H, et al.: Long-term evaluation of combined prolonged-release oxycodone and naloxone in patients with moderate-to-severe chronic pain: Pooled analysis of extension phases of two phase III trials. Neurogastroenterol Motil 2014;26:1792-1801.

8. Cloutier C, Taliano J, O'Mahony W, et al.: Controlledrelease oxycodone and naloxone in the treatment of chronic low back pain: A placebo-controlled, randomized study. Pain Res Manag 2013;18:75-82.

9. Dupoiron D, Stachowiak A, Loewenstein O, et al.: A phase III randomized controlled study on the efficacy and improved bowel function of prolonged-release (PR) oxycodone-naloxone (up to 160/80 mg daily) vs oxycodone PR. Eur J Pain 2017;21: $1528-1537$.

10. Koopmans G, Simpson K, De Andres J, et al.: Fixed ratio (2: 1) prolonged-release oxycodone/naloxone combination improves bowel function in patients with moderate-to-severe pain and opioid-induced constipation refractory to at least two classes of laxatives. Curr Med Res Opin 2014;30:2389-2396.

11. Lowenstein O, Leyendecker P, Hopp M, et al.: Combined prolonged-release oxycodone and naloxone improves bowel function in patients receiving opioids for moderate-to-severe non-malignant chronic pain: A randomised controlled trial. Expert Opin Pharmacother 2009;10:531-543.

12. Lowenstein O, Leyendecker P, Lux EA, et al.: Efficacy and safety of combined prolonged-release oxycodone and naloxone in the management of moderate/severe chronic nonmalignant pain: Results of a prospectively designed pooled analysis of two randomised, double-blind clinical trials. BMC Clin Pharmacol 2010;10:12.

13. Meissner W, Leyendecker P, Mueller-Lissner S, et al.: A randomised controlled trial with prolonged-release oral oxycodone and naloxone to prevent and reverse opioidinduced constipation. Eur J Pain 2009;13:56-64.

14. Poelaert J, Koopmans-Klein G, Dioh A, et al.: Treatment with prolonged-release oxycodone/naloxone improves pain relief and opioid-induced constipation compared with prolongedrelease oxycodone in patients with chronic severe pain and laxative-refractory constipation. Clin Ther 2015;37:784-792.

15. Simpson K, Leyendecker P, Hopp M, et al.: Fixed-ratio combination oxycodone/naloxone compared with oxycodone alone for the relief of opioid-induced constipation in moderate-to-severe noncancer pain. Curr Med Res Opin 2008;24:3503-3512.

16. van Dongen VC, Vanelderen PJ, Koopmans-Klein G, et al.: Patient preference with respect to QoL and reduction in opioid-induced constipation (OIC) after treatment with prolonged-release (PR) oxycodone/naloxone compared with previous analgesic therapy [PREFER study]. Int J Clin Pract 2014;68:1364-1375.
17. Kim ES: Oxycodone/naloxone prolonged release: A review in severe chronic pain. Clin Drug Investig 2017;37:1191-1201.

18. Pond SM, Tozer TN: First-pass elimination. Basic concepts and clinical consequences. Clin Pharmacokinet 1984;9:1-25.

19. Wong A, Macleod D, Robinson J, et al.: Oxycodone/naloxone preparation can cause acute withdrawal symptoms when misused parenterally or taken orally. Clin Toxicol (Phila) 2015;53:815-818.

20. Franklin AE, Lovell MR, Boyle F: A case of opioid toxicity on conversion from extended-release oxycodone and naloxone to extended-release oxycodone in a patient with liver dysfunction. J Pain Symptom Manage 2017;53:e1-e2.

21. Oxycodone-with-naloxone controlled-release tablets (Targin) for chronic severe pain. https://www.nps.org.au/radar/ articles/oxycodone-with-naloxone-controlled-release-tabletstargin-for-chronic-severe-pain\#article. 2011. (Last Accessed July 7, 2019).

22. Currow DC, Rowett D, Doogue M, et al.: An international initiative to create a collaborative for pharmacovigilance in hospice and palliative care clinical practice. J Palliat Med 2012;15:282-286.

23. Digges M, Hussein A, Wilcock A, et al.: Pharmacovigilance in hospice/palliative care: Net effect of haloperidol for nausea or vomiting. J Palliat Med 2018;21:37-43.

24. Hatano Y, Moroni M, Wilcock A, et al.: Pharmacovigilance in hospice/palliative care: The net immediate and shortterm effects of dexamethasone for anorexia. BMJ Support 2016;6:331-337.

25. Sanderson C, Quinn SJ, Agar M, et al.: Pharmacovigilance in hospice/palliative care: Net effect of gabapentin for neuropathic pain. BMJ Support 2015;5:273-280.

26. Sanderson C, Quinn SJ, Agar M, et al.: Pharmacovigilance in hospice/palliative care: Net effect of pregabalin for neuropathic pain. BMJ Support 2016;6:323-330.

27. Anon: Common Terminology Criteria for Adverse Events (CTCAE) Version 5 2017. https://ctep.cancer.gov/protocold evelopment/electronic_applications/ctc.htm\#ctc_50.2019. (Last accessed March 31, 2019).

28. Caraceni A, Hanks G, Kaasa S, et al.: Use of opioid analgesics in the treatment of cancer pain: Evidence-based recommendations from the EAPC. Lancet Oncol 2012;13:e58-e68.

29. Dumas EO, Pollack GM: Opioid tolerance development: A pharmacokinetic/pharmacodynamic perspective. AAPS J 2008;10:537-551.

30. Mercadante S, Caraceni A: Conversion ratios for opioid switching in the treatment of cancer pain: A systematic review. Palliat Med 2011;25:504-515.

31. Webster L, Fine P: Overdose deaths demand a new paradigm for opioid rotation. Pain Med 2012;13:571-574.

32. Dzierżanowski T, Ciałkowska-Rysz A: Behavioral risk factors of constipation in palliative care patients. Support Care Cancer 2015;23:1787-1793.

Address for correspondence: Katherine Clark, MBBS, MMED, PhD, FRACP, FAChPM Department of palliative Care Northern Sydney Local Health District Cancer and Palliative Care Network Reserve Road St. Leonards 2065 Australia

E-mail: katherine.clark@health.nsw.gov.au 